PROCEEDINGS OF THE

AMERICAN MATHEMATICAL SOCIETY

Volume 135, Number 12, December 2007, Pages 4039-4044

S 0002-9939(07)08952-6

Article electronically published on August 30, 2007

\title{
EXTREMAL PSEUDOCOMPACT ABELIAN GROUPS ARE COMPACT METRIZABLE
}

\author{
W. W. COMFORT AND JAN VAN MILL
}

(Communicated by Alexander N. Dranishnikov)

\begin{abstract}
Every pseudocompact Abelian group of uncountable weight has both a proper dense pseudocompact subgroup and a strictly finer pseudocompact group topology.
\end{abstract}

\section{INTRODUCTION}

All topological groups here are assumed to satisfy the Hausdorff separation axiom. A pseudocompact group $G$ is said to be $r$-extremal [resp. s-extremal] if $G$ admits no strictly finer pseudocompact group topology [resp. $G$ has no proper dense pseudocompact subgroup]. Early formulations of these notions appeared in [6], 7. From the fact that a pseudocompact space of countable weight is compact and metrizable it follows readily (as in [7, 2.3]) that every pseudocompact group of countable weight is both $r$-extremal and $s$-extremal. It is natural to ask whether there are extremal pseudocompact groups of uncountable weight. This question has generated much attention during the last two decades. See [1, [12] and [10] for more information. An affirmative answer was given in 7 for zero-dimensional Abelian groups. In 2 it was shown that no pseudocompact Abelian group of cardinality greater than $\mathfrak{c}$ is $s$-extremal. For partial answers in the class of connected groups, see for example [3, 12] and 1].

The aim of this paper is to answer the question for Abelian groups.

Theorem 1.1. A pseudocompact Abelian group of uncountable weight is neither r-extremal nor s-extremal.

We keep this presentation short by invoking several essential results established in the literature. We plan in [5] to present a polished, complete and self-contained proof of Theorem 1.1.

We announced our results at the annual meeting of the American Mathematical Society in January, 2006 [4].

\section{Preliminaries}

In this section we fix notation and we cite the results we need from the literature.

Received by the editors November 20, 2005 and, in revised form, September 2, 2006.

2000 Mathematics Subject Classification. Primary 22A05, 22B05.

Key words and phrases. Pseudocompact topological group, extremal topological group, proper dense pseudocompact subgroup, Abelian.

(C)2007 American Mathematical Society 
The symbol $w X$ denotes the weight of a topological space $X$. A subspace of a space $X$ is $G_{\delta}$-dense in $X$ if it meets every nonempty $G_{\delta}$-subset of $X$. If $X$ is a set and $\kappa$ a cardinal number, then $[X]^{\leq \kappa}$ denotes $\{A \subseteq X:|A| \leq \kappa\}$.

For Abelian groups we use additive notation. Let $G$ be an Abelian group. If $A \subseteq G$, then $\langle\langle A\rangle\rangle$ denotes the subgroup of $G$ generated by $A$. A subset $X$ of $G$ is called independent if for every $x \in X$ we have $\langle\langle\{x\}\rangle \cap \cap\langle\langle X \backslash\{x\}\rangle\rangle=\{0\}$. If $A$ is a subgroup of $G$, then a subset $X$ of $G$ is said to be independent over $A$ if it is independent and $\langle\langle X\rangle\rangle \cap A=\{0\}$. The cardinality of a maximal independent set of elements of infinite order is called the torsion-free rank of $G$, here denoted $r_{0}(G)$. It is known that $r_{0}(G)$ is an invariant of $G$, i.e., all such maximal independent subsets of $G$ have the same cardinality. It is clear that if $h: G \rightarrow H$ is a surjective homomorphism, then $r_{0}(H) \leq r_{0}(G)$. See [11, pp. 85-86] for additional details. The torsion subgroup of an Abelian group $G$ is denoted by $t G$.

If $G$ is a (not necessarily Abelian) totally bounded group, then $\bar{G}$ denotes its (compact) Weil completion. It was shown in [8 that a topological group $G$ is pseudocompact if and only if it is $G_{\delta}$-dense in $\bar{G}$. Hence a dense subgroup of a pseudocompact group $G$ is pseudocompact if and only if it is $G_{\delta}$-dense in $G$.

Let $G$ be a topological group. Then

$$
\Lambda(G)=\left\{N \subseteq G: N \text { is a closed, normal, } G_{\delta^{-}} \text {-subgroup of } G\right\} .
$$

Now we collect some information needed later in our proof of the main result.

Theorem 2.1. Let $G$ be a pseudocompact group such that $w G>\omega$, and let $N \in$ $\Lambda(G)$. Then

(a) [9, 3.3] $G / N$ is compact and metrizable,

(b) [7, 6.2] $N$ is pseudocompact, and

(c) [3, 2.7] $w N=w G$.

Lemma 2.2 ([3, 2.13(b),(c)]). Let $G$ be a pseudocompact group and let $G=$ $\bigcup_{n<\omega} A_{n}$, where each $A_{n}$ is a subgroup of $G$. Then there exist $N \in \Lambda(G)$ and $n<\omega$ such that $A_{n} \cap N$ is $G_{\delta}$-dense in $N$.

Theorem 2.3 (11,4.4], [12, 3.7.1]). Let $G$ be a pseudocompact Abelian group . If $G$ contains a proper, dense pseudocompact subgroup $H$ such that $G / H$ can be mapped homomorphically onto some nondegenerate compact group, then $G$ is not $r$-extremal.

Theorem 2.4 ([1, 5.7], [12, 6.4.2]). Let $G$ be a pseudocompact Abelian group of uncountable weight. If there exists $N \in \Lambda(G)$ such that no connected $M \in \Lambda(G)$ is contained in $N$, then $G$ is neither $r$-extremal nor s-extremal.

Theorem 2.5 ([2, 4.5], [1, 5.10], [12, 7.3]). Let $G$ be a pseudocompact Abelian group of uncountable weight such that $r_{0}(G)>\mathfrak{c}$. Then $G$ is neither $r$-extremal nor s-extremal.

\section{LEMMAS}

In this section we collect some simple results to be used later. The technique used in the proof of Lemma 3.1 is well-known, and was used in many earlier results. See e.g., [3, 2, 12, 1]. For the benefit of the reader we provide the (simple) details. (Note added September 15, 2006. The referee has pointed out that a proof of Lemma 3.1 is also available in the preprint [10].) 
Lemma 3.1. Let $G$ be a pseudocompact Abelian group, and let $A$ be a $G_{\delta}$-dense subgroup of some $N \in \Lambda(G)$ such that $r_{0}(N / A) \geq \mathfrak{c}$. Then $G$ contains a $G_{\delta}$-dense subgroup $H$ such that $r_{0}(G / H) \geq \mathfrak{c}$.

Proof. The conditions imply that there is a subset $X$ of $N \backslash A$ of elements of infinite order such that $|X|=\mathfrak{c}$ and $X$ is independent over $A$. Split $X$ into two disjoint sets $X_{0}$ and $X_{1}$, each of cardinality $\mathfrak{c}$.

By Theorem 2.1(a), the number of cosets of $N$ in $G$ is at most $\mathfrak{c}$. (In fact, either $|G / N|<\omega$ or $|G / N|=$ c.) Let $\left\{a_{\alpha}+N: \alpha<\lambda\right\}$ be a faithful enumeration of $G / N$. We assume without loss of generality that $a_{0}=0$. By recursion on $\alpha<\lambda$ we will choose $x_{\alpha} \in X_{0} \cup\{0\}$ such that

$$
\left.\left\langle\left\langle X_{1}\right\rangle\right\rangle \cap\left(\left\langle\left\{a_{\beta}+x_{\beta}: \beta \leq \alpha\right\}\right\rangle\right\rangle+A\right)=\{0\} .
$$

Let $x_{0}=0$. Let $\alpha<\lambda$ and suppose that $x_{\beta}$ has been defined for all $\beta<\alpha$. Put $B_{\alpha}=\left\langle\left\langle\left\{a_{\beta}+x_{\beta}: \beta<\alpha\right\}\right\rangle\right\rangle$. Then $\left|B_{\alpha}\right|<\mathfrak{c}$ and $\left\langle\left\langle X_{1}\right\rangle\right\rangle \cap\left(B_{\alpha}+A\right)=\{0\}$. Suppose that for every $x \in X_{0}$ we have that

$$
\left\langle\left\langle X_{1}\right\rangle\right\rangle \cap\left(\left\langle\left\langle B_{\alpha} \cup\left\{a_{\alpha}+x\right\}\right\rangle\right\rangle+A\right) \neq\{0\} .
$$

Then for every $x \in X_{0}$ there exist $b_{x} \in B_{\alpha}, n_{x} \in \mathbb{Z}, p_{x} \in A$ and $q_{x} \in\left\langle\left\langle X_{1}\right\rangle\right\rangle \backslash\{0\}$ such that

$$
q_{x}=b_{x}+n_{x}\left(a_{\alpha}+x\right)+p_{x} .
$$

Note (since $q_{x} \notin B_{\alpha}+A$ ) that no $n_{x}$ is equal 0 . Since $\left|X_{0}\right|=\mathfrak{c}$, there are distinct $x, y \in X_{0}, n \in \mathbb{Z} \backslash\{0\}$ and $b \in B_{\alpha}$ such that $n=n_{x}=n_{y}$ and $b=b_{x}=b_{y}$. But then by subtracting the equation $(\dagger)$ for $x$ and $y$, we get

$$
n(x-y)=q_{x}-q_{y}+p_{y}-p_{x} \in\left\langle\left\langle X_{1}\right\rangle\right\rangle+A,
$$

which contradicts the independence of $X$ over $A$. This completes the transfinite recursion.

Now put $B=\bigcup_{\alpha<\lambda} B_{\alpha}$. Then $\left\langle\left\langle X_{1}\right\rangle\right\rangle \cap(B+A)=\{0\}$, hence $r_{0}(G /(B+A)) \geq$ $\left|X_{1}\right|=\mathfrak{c}$. It is clear that $B+A$ is $G_{\delta^{-}}$-dense in $G$.

Lemma 3.2. Let $\kappa$ be an infinite cardinal. Suppose that $\mathcal{A}$ is a family of subsets of $2^{\kappa}$ with the following properties:

(1) if $\mathcal{B} \in[\mathcal{A}]^{\leq \kappa}$, then $\bigcap \mathcal{B} \in \mathcal{A}$, and

(2) each element of $\mathcal{A}$ has cardinality $2^{\kappa}$.

Then there is a countably infinite family $\mathcal{B}$ of subsets of $2^{\kappa}$ such that

(i) $\mathcal{B}$ is pairwise disjoint, and

(ii) if $A \in \mathcal{A}$ and $B \in \mathcal{B}$, then $|A \cap B|=2^{\kappa}$.

Proof. We give $2^{\kappa}$ the standard Tychonov product topology. Let $\mathcal{V}$ be the collection of all nonempty clopen subsets $V$ of $2^{\kappa}$ for which there is an element $A(V) \in \mathcal{A}$ such that $|V \cap A(V)|<2^{\kappa}$. Clearly, $|\mathcal{V}| \leq \kappa$. Let $\mathcal{D}=\{A(V): V \in \mathcal{V}\}, Y=\bigcap \mathcal{D}$, and $\widetilde{V}=\bigcup \mathcal{V}$. Now $|V \cap Y| \leq|V \cap A(V)|<2^{\kappa}$ for every $V \in \mathcal{V}$, so

$$
|\widetilde{V} \cap Y|<2^{\kappa}
$$

since $2^{\kappa}$ has cofinality at least $\kappa^{+}$. Then $|Y|=2^{\kappa}$ by (1) and (2), hence $|Y \backslash \widetilde{V}|=$ $2^{\kappa}$. There is consequently a countably infinite pairwise disjoint family $\mathcal{B}$ of clopen subsets of $2^{\kappa}$ such that $B \cap(Y \backslash \widetilde{V}) \neq \emptyset$ for every $B \in \mathcal{B}$. To see that $\mathcal{B}$ is as 
required, pick arbitrary $B \in \mathcal{B}$ and $A \in \mathcal{A}$. If $|B \cap A|<2^{\kappa}$, then $B \in \mathcal{V}$ and hence $B \subseteq \widetilde{V}$, which contradicts the fact that $B \cap(Y \backslash \widetilde{V}) \neq \emptyset$.

Remark 3.3. The inclusion $\bigcup \mathcal{B} \subseteq 2^{\kappa}$ is necessarily proper (since $2^{\kappa}$ is compact). Replacing any one element of $\mathcal{B}$ by the complement in $2^{\kappa}$ of the union of the remaining elements, we may hence assume without loss of generality that $\mathcal{B}$ is a partition.

\section{Proof of Theorem 1.1}

We now present the proof of our main result. By Theorem 2.5, it suffices to consider groups $G$ of torsion-free rank at most c. Furthermore, by Theorem 2.4 we may assume that every $N \in \Lambda(G)$ contains a connected $M \in \Lambda(G)$. Henceforth, let $G$ be a pseudocompact Abelian group of uncountable weight satisfying those two conditions.

Lemma 4.1. If $H$ is a nontrivial connected subgroup of $G$, then $r_{0}(H)=\mathfrak{c}$.

Proof. It is clear that $r_{0}(H) \leq \mathfrak{c}$. Let $0 \neq x \in H$ and let $h$ be a continuous homomorphism from $H$ to $\mathbb{T}$ such that $h(x) \neq h(0)$. Then $h(H)=\mathbb{T}$ since $H$ (and hence $h(H))$ is connected. It follows that $\mathfrak{c} \geq r_{0}(H) \geq r_{0}(\mathbb{T})=\mathfrak{c}$, as asserted.

Since $G \in \Lambda(G)$, there is a connected $C \in \Lambda(G)$. Hence $r_{0}(C)=\mathfrak{c}$ by Theorem 2.1(b),(c) and Lemma 4.1.

Let $\bar{C}$ be the closure of $C$ in $\bar{G}$. Then $\bar{C}$ is a compact, connected group, hence is divisible [13, Theorem 24.25]. By [13, Theorem A.14] or [11, Theorem 23.1], there is a cardinal number $\lambda$ such that $\bar{C}$ is (algebraically) isomorphic to

$$
\bigoplus_{\alpha<\lambda} \mathbb{Q}_{\alpha} \oplus t \bar{C}
$$

where each $\mathbb{Q}_{\alpha}$ is a copy of the group of rational numbers $\mathbb{Q}$. Then $\bar{C} / t \bar{C}=$ $\bigoplus_{\alpha<\lambda} \mathbb{Q}_{\alpha}$. Let $\pi: \bar{C} \rightarrow \bigoplus_{\alpha<\lambda} \mathbb{Q}_{\alpha}$ be the natural homomorphism. For $x \in \bar{C}$ let $S(x)=\left\{\alpha<\lambda: \pi(x)_{\alpha} \neq 0_{\alpha}\right\}$, and for $E \subseteq \bar{C}$ let $S(E)=\bigcup_{x \in E} S(x)$.

Lemma 4.2. If $N \in \Lambda(C)$, then $|S(\pi(N))|=\mathfrak{c}$.

Proof. Since $N \in \Lambda(G)$, we may assume without loss of generality that $N$ is connected. Moreover, $N$ is nontrivial by Theorem 2.1(c). So $r_{0}(N)=\mathfrak{c}$ by Lemma 4.1. That $|S(\pi(N))|=\mathfrak{c}$ is then clear.

Writing $S=S(\pi(C))$, we have $|S|=\mathfrak{c}$. Hence $\lambda \geq \mathfrak{c}$, and

$$
C \subseteq \bigoplus_{\alpha \in S} \mathbb{Q}_{\alpha} \oplus \bigoplus_{\alpha \notin S}\left\{0_{\alpha}\right\} \oplus t \bar{C} .
$$

For every $\beta \in S$, let $\rho_{\beta}: \bigoplus_{\alpha \in S} \mathbb{Q}_{\alpha} \rightarrow \mathbb{Q}_{\beta}$ be the projection.

For every nonempty $A \subseteq S$, put

$$
G(A)=C \cap\left(\bigoplus_{\alpha \in A} \mathbb{Q}_{\alpha} \oplus \bigoplus_{\alpha \notin A}\left\{0_{\alpha}\right\} \oplus t \bar{C}\right),
$$

and let

$$
\mathcal{A}=\{A \subseteq S: \text { there is } N \in \Lambda(C) \text { such that } N \subseteq G(A)\} .
$$

Lemma 4.3. $\mathcal{A}$ is closed under countable intersections, and every $A \in \mathcal{A}$ has size c. 
Proof. That $\mathcal{A}$ is closed under countable intersections is clear, since if $\mathcal{B}$ is any family of subsets of $S$, then

$$
\bigcap_{B \in \mathcal{B}} G(B)=G(\bigcap \mathcal{B})
$$

and $\Lambda(G)$ is closed under countable intersections.

Now take an arbitrary $A \in \mathcal{A}$. We want to prove that $|A|=\mathfrak{c}$. Take $N \in \Lambda(C)$ such that $N \subseteq G(A)$. Then $\pi(N) \subseteq A$, so $\mathfrak{c}=|S| \geq|A| \geq|\pi(N)|=\mathfrak{c}$ by Lemma 4.2 .

By Lemma 3.2 and Remark 3.3, there consequently is a (faithfully indexed) partition $\mathcal{B}=\left\{B_{n}: n<\omega\right\}$ of $S$ such that $\left|B_{n} \cap A\right|=\mathfrak{c}$ for each $B_{n} \in \mathcal{B}, A \in \mathcal{A}$. For every $n<\omega$, let

$$
V_{n}=G\left(\bigcup_{i \leq n} B_{i}\right) .
$$

Then $V_{0} \subseteq V_{1} \subseteq \cdots \subseteq V_{n} \subseteq \cdots$, and $C=\bigcup_{n<\omega} V_{n}$. By Theorem 2.1(b) and Lemma 2.2, there exist $N \in \Lambda(C)$ and $m<\omega$ such that $H:=V_{m} \cap N$ is $G_{\delta^{-}}$ dense in $N$. We may assume without loss of generality that $m=0$, i.e., that $V_{m}=V_{0}=G\left(B_{0}\right)$.

Lemma 4.4. $r_{0}(N / H) \geq \mathfrak{c}$.

Proof. We will prove that there is a subset $X$ of $N$ of cardinality $\mathfrak{c}$ such that

(1) each $x \in X$ has infinite order,

(2) $X$ is independent,

(3) $\langle\langle X\rangle\rangle \cap H=\{0\}$

(hence $\langle\langle X\rangle\rangle$ is isomorphic to $\bigoplus_{\alpha<c} \mathbb{Z}_{\alpha}$, where each $\mathbb{Z}_{\alpha}$ is a copy of the group of integers $\mathbb{Z})$. Choose $x_{0} \in N \backslash G\left(B_{0}\right)$ and define $W_{0}=B_{0}$. Let $0<\alpha<\mathfrak{c}$ and suppose that $x_{\beta}$ and $W_{\beta}$ have been defined for all $\beta<\alpha$. Then, set

$$
W_{\alpha}=B_{0} \cup \bigcup_{\beta<\alpha} S\left(x_{\beta}\right),
$$

and observe that

$$
\left|B_{1} \cap W_{\alpha}\right|=\left|B_{1} \cap \bigcup_{\beta<\alpha} S\left(x_{\beta}\right)\right|<\mathfrak{c} .
$$

Hence $W_{\alpha} \notin \mathcal{A}$, since $B_{1}$ meets every element of $\mathcal{A}$ in a set of size $\mathfrak{c}$, which means that $N \nsubseteq G\left(W_{\alpha}\right)$; let $x_{\alpha}$ be any point in $N \backslash G\left(W_{\alpha}\right)$. This completes the transfinite construction.

We claim that $X=\left\{x_{\alpha}: \alpha<\mathfrak{c}\right\}$ satisfies (1), (2) and (3). To prove this, let $\alpha<\mathfrak{c}$, and let $n \in \mathbb{Z} \backslash\{0\}$ be arbitrary. By construction we have

$$
x_{\alpha} \notin G\left(B_{0} \cup \bigcup_{\beta<\alpha} S\left(x_{\beta}\right)\right),
$$

so $S\left(x_{\alpha}\right) \nsubseteq B_{0} \cup \bigcup_{\beta<\alpha} S\left(x_{\beta}\right)$; let $\gamma \in S\left(x_{\alpha}\right)$ witness that relation. Then $\rho_{\gamma}\left(\pi\left(x_{\alpha}\right)\right) \neq$ 0 and $\rho_{\gamma}\left(\pi\left(x_{\beta}+h\right)\right)=0$ for every $\beta<\alpha$ and $h \in H$. Then clearly $x_{\alpha}$ has infinite order, and

as required.

$$
n x_{\alpha} \notin\left\langle\left\langle\left\{x_{\beta}: \beta<\alpha\right\}\right\rangle\right\rangle+H,
$$


Now we complete the proof of Theorem 1.1. From Lemmas 3.1 and 4.4 we conclude that $G$ has a proper dense pseudocompact subgroup $H$ such that $r_{0}(G / H) \geq$ c. This proves that $G$ is not $s$-extremal. To see that $G$ is not $r$-extremal, note first that $r_{0}(G / H) \geq \mathfrak{c}$, so $G / H$ contains a subgroup isomorphic to $\bigoplus_{\alpha<\mathfrak{c}} \mathbb{Z}_{\alpha}$. The latter can be mapped homomorphically onto $\mathbb{T}$, and since homomorphisms into a divisible group always extend by [13, Theorem A.7], we are done by Theorem 2.3.

\section{REFERENCES}

[1] W. W. Comfort and J. Galindo, Extremal pseudocompact topological groups, J. Pure Appl. Algebra 197 (2005), 59-81. MR2123980 (2006a:22001)

[2] W. W. Comfort, H. Gladdines, and J. van Mill, Proper pseudocompact subgroups of pseudocompact abelian groups, Annals of the New York Ac. Sci. 728 (1994), 237-247. MR 1467777 (99g:22005)

[3] W. W. Comfort and J. van Mill, Concerning connected, pseudocompact Abelian groups, Top. Appl. 33 (1989), 21-45. MR.1020981 (90k:54002)

[4] W. W. Comfort and J. van Mill, Extremal pseudocompact Abelian groups are compact metric, Abstracts Amer. Math. Soc. 27 (2006), p. 78 (Abstract \#1014-22-958).

[5] W. W. Comfort and J. van Mill, Extremal pseudocompact abelian groups: A brief exposé, in preparation.

[6] W. W. Comfort and L. C. Robertson, Proper pseudocompact extensions of compact abelian group topologies, Proc. Amer. Math. Soc. 86 (1982), 173-178. MR663891 (83k:22011)

[7] W. W. Comfort and Lewis C. Robertson, Extremal phenomena in certain classes of totally bounded groups, Dissertationes Math. (Rozprawy Mat.) 272 (1988), 1-42. MR959432 (89i:22001)

[8] W. W. Comfort and K. A. Ross, Pseudocompactness and uniform continuity in topological groups, Pac. J. Math. 16 (1966), 483-496. MR0207886 (34:7699)

[9] W. W. Comfort and T. Soundararajan, Pseudocompact group topologies and totally dense subgroups, Pacific J. Math. 100 (1982), 61-84. MR661441 (83m:22008)

[10] D. Dikranjan, G. Bruno, and C. Milan, Weakly metrizable pseudocompact groups, to appear in Appl. Gen. Top.

[11] L. Fuchs, Infinite abelian groups. Vol. I, Academic Press, New York, 1970. MR0255673 $(41: 333)$

[12] J. Galindo, Dense pseudocompact subgroups and finer pseudocompact group topologies, Sci. Math. Jpn. 55 (2002), 627-640. MR.1901051 (2003b:54041)

[13] E. Hewitt and K. A. Ross, Abstract harmonic analysis I, Springer-Verlag, New YorkHeidelberg-Berlin, 1979. MR551496 (81k:43001)

Department of Mathematics, Wesleyan University, Middletown, Connecticut 06459

E-mail address: wcomfort@wesleyan.edu

$U R L:$ http://wcomfort.web.wesleyan.edu

Faculteit Exacte Wetenschappen, Afdeling Wiskunde, Vrije Universiteit, De BoelelaAn 1081A, 1081 HV Amsterdam, The Netherlands

E-mail address: vanmill@cs.vu.nl

$U R L:$ http://www.few.vu.nl/ vanmill 